original lists when doubts arose. Ultimately, however, the editors found it impossible to take personal responsibility for every date, and this responsibility rests, as it always has rested, on the laboratories. Their cooperation has been exemplary, despite the numerous changes of personnel, organizational structure, and laboratory practice that have occurred since 1950. That all concerned will have avoided all mistakes is too much to expect, and we trust that users of the Index will forgive the mistakes they find, and call them to the editors' attention when they find them.

The generous support of the National Science Foundation, through Grant GN-396, is gratefully acknowledged.

Edward S. Deevey, JR. RichaRd Foster Flint IRVING Rouse

\title{
Published Reference Code
}

1 Deevey et al., 1954, Natl. Acad. Sci. Proc., v. 40, p. 285-288.

Yale I, 1953, Science, v. 118, p. 1-6.

Yale II, 1955, Science, v. 122, p. 954-960.

Yale III, 1957, Science, v. 126, p. 908-919.

Yale IV, 1959, Radiocarbon Suppl., v. 1 p. 144-172.

Yale V, 1960, Radiocarbon Suppl., v. 2, p. 49-61.

Yale VI, 1961, Radiocarbon, v. 3, p. 126-140.

Yale VII, 1962, Radiocarbon, v. 4, p. 250-262.

Lamont I, 1951, Science, v. 114, p. 565-568.

Lamont II, 1952, Science, v. 116, p. 490-414.

Lamont III, 1956, Science, v. 124, p. 154-165.

2 Lamont IV, 1957, Science, v. 126, p. 1324-1334.

Lamont V, 1959, Radiocarbon Suppl., v. 1. p. 1-28.

Lamont VI, 1959, Radiocarbon Suppl., v. 1, p. 111-132.

Lamont VII, 1961, Radiocarbon, v. 3, p. 141-175.

Lamont VIII, 1961, Radiocarbon, v. 3, p. 176-204.

USGS I, 1954, Science, v. 120, p. 467-473.

USGS II, 1955, Science, v. 121, p. 481-488.

USGS III, 1956, Science, v. 123, p. 442-448.

USGS IV, 1958, Science, v. 127, p. 1476-1487.

USGS V, 1960, Radiocarbon Suppl., v. 2, p. 129-185.

USGS VI, 1961, Radiocarbon, v. 3, p. 86-97.

Michigan I, 1956, Science, v. 124, p. 664-672.

Michigan II, 1958, Science, v. 127, p. 1098-1105.

Michigan III, 1958, Science, v. 128, p. 1117-1123.

Michigan IV, 1959, Radiocarbon Suppl., v. 1, p. 173-198.

Michigan V, 1960, Radiocarbon Suppl., v. 2, p. 31-48.

Michigan VI, 1961, Radiocarbon, v. 3, p. 105-125.

Michigan VII, 1962, Radiocarbon, v. 4, p. 183-203.

Heidelberg I, 1957, Science, v. 126, p. 194-199.

Copenhagen I, 1953, Science, v. 118, p. 6-9.

Iversen, 1953, Science, v. 118, p. 9-11.

33 Copenhagen II, 1956, Science, v. 124, p. 879-881.

34 Vinogradov, A. P., 1962, p. 86-88 in "Radioisotopes in the physical sciences and industry, Internat. Atom. Energy Agency, Vienna, 1962. 
35 Groningen II, 1958, Science, v. 127, p. 129-137.

36 Groningen III, 1958, Science, v. 128, p. 1550-1556.

37 Stockholm I, 1957, Science, v. 126, p. 493-497.

38 Stockholm II, 1959, Radiocarbon Suppl., v. 1, p. 35-44.

39 Stockholm III, 1960, Radiocarbon Suppl., v. 2, p. 186-196.

40 Stockholm IV, 1962, Radiocarbon, v. 4, p. 115-136.

41 Trondheim I, 1959, Radiocarbon Suppl., v. 1, p. 76-80.

42 Trondheim II, 1960, Radiocarbon Suppl., v. 2, p. 82-96.

43 Trondheim III, 1962, Radiocarbon, v. 4, p. 160-182.

44. Cambridge I, 1959, Radiocarbon Suppl., v. 1, p. 63-75.

45 Cambridge II, 1960, Radiocarbon Suppl., v. 2, p. 62-72.

46 Cambridge III, 1961, Radivearbon, v. 3, p. 60-76.

47 Cambridge IV, 1961, Radiocarbon, v. 3, p. 77-80.

48 Cambridge V, 1962, Radiocarbon, v. 4, p. 57-70.

49 Humble I, 1957, Science, v. 125, p. 147-150.

50 Humble II, 1957, Science, v. 125, p. 919-923.

51 Wise and Shutler, 1958, Science, v. 127, p. 72-74.

52 Arizona II, 1959, Radiocarbon Suppl., v. 1, p. 59-62.

53 Arizona III, 1962, Radiocarbon, v. 4, p. 239-249.

54. British Museum I, 1959, Radiocarbon Suppl., v. 1, p. 81-86.

55 British Museum II, 1960, Radiocarbon Suppl., v. 2, p. 26-30.

56 British Museum III, 1961, Radiocarbon, v. 3, p. 39-45.

57 Pringle et al., 1957, Science, v. 125, p. 69-70.

58 Santa Rosa Island I, 1956, Santa Barbara Mus. Nat. History, Dept. Anthropology, Bull. 2, p. 1-10.

59 Ralph and Stuckenrath, 1960, Nature, v. 188, p. 185-187.

60 Pennsylvania I, 1955, Science, v. 121, p. 149-151.

61 Pennsylvania II, 1955, Science, v. 122, p. 921-922.

62 Pennsylvania III, 1959, Radiocarbon Suppl., v. 1, p. 45-58.

63 Pennsylvania IV, 1961, Radiocarbon, v. 3, p. 4-14.

64. Pennsylvania V, 1962, Radiocarbon, v. 4, p. 144-159.

64a Kohler and Ralph, 1961, Am. J. Archaeol., v. 65, p. 357-367.

65 Uppsala I, 1959, Radiocarbon Suppl., v. 1, p. 87-102.

66 Uppsala II, 1960, Radiocarbon Suppl., v. 2, p. 112-128.

67 Uppsala III, 1961, Radiocarbon, v. 3, p. 81-85.

68 Ferrara et al., 1959, Radiocarbon Suppl., v. 1, p. 103-110.

69 Pisa II, 1961, Radiocarbon, v. 3, p. 99-104.

70 Bern I, 1959, Radiocarbon Suppl., v. 1, p. 133-143.

71 Bern II, 1961, Radiocarbon, v. 3, p. 15-25.

72 Socony Mobil I, 1960, Radiocarbon Suppl., v. 2, p. 97-111.

3 La Jolla I, 1960, Radiocarbon Suppl., v. 2, p. 197-223.

La Jolla II, 1962, Radiocarbon, v. 4, p. 204-238.

Isotopes I, 1961, Radiocarbon, v. 3, p. 47-59.

Isotopes II, 1962, Radiocarbon, v. 4, p. 35-42.

Dublin I, 1961, Radiocarbon, v. 3, p. 26-38.

Washington I, 1962, Radiocarbon, v. 4, p. 1-12.

Geological Survey of Canada I, 1962, Radiocarbon, v. 4, p. 13-26.

Texas I, 1962, Radiocarbon, v. 4, p. 43-50.

Miami I, 1962, Radiocarbon, v. 4, p. 51-56

Geological Survey of Finland I, 1962, Radiocarbon, v. 4, p. 81-83.

Gakushuin I, 1962, Radiocarbon, v. 4, p. 84-94.

Louvain I, 1962, Radiocarbon, v. 4, p. 95-99.

Hannover I, 1962, Radiocarbon, v. 4, p. 100-108.

Hannover II, 1962, Radiocarbon, v. 4, p. 137-143.

UCLA I, 1962, Radiocarbon, v. 4, p. 109-114.

Vinogradov, A. P., and others, 1962, Determination of absolute age according to $\mathrm{C}^{14}$ :

Akad. Nauk, USS, Geokhimiya, 1962, no. 5, p. 387-402.

89 Saskatchewan II, 1960, Radiocarbon Suppl., v. 2, p. 73-81.

90 Saskatchewan III, 1962, Radiocarbon, v. 4, p. 71-80.

91 Copenhagen III, 1960, Radiocarbon Suppl., v. 2, p. 5-11.

92 Copenhagen IV, 1960, Radiocarbon Suppl., v. 2, p. 12-25.

93 Copenhagen V, 1962, Radiocarbon, v. 4, p. 27-34.

94. Heusser, 1959, Radiocarbon Suppl., v. 1, p. 29-34. 
95 Libby, 1955, Radiocarbon Dating, 2nd ed.: Chicago, Univ. Chicago Press

96 DeVries and Barendsen, 1954, Nature, v. 174, p. 1138-1146.

97 Ballario et al., 1955, Science, v. 121, p. 409-412.

97a Rome I, 1957, La Ricerca Scientifica, C. N. R., Roma, v. 27, p. 2677-2680

98 Zeuner, F., 1956, Antiquity, v. 30, p. 195-196.

98a Zeuner, F., 1955, Inst. Archeol., Univ. London, 11th Ann. Rep., No. P 1-8.

99 Vaughan, D. E. and Zeuner, F., n.d., paper Davy-Faraday Laboratory, Royal Institution of London, p. 1-3.

100 Perquis et al., 1956, Commissariat a l'Energie Atomique Bull. Inf. Sci. Tech., no. 11, p. 1-7.

101 Delibrias, G., and Perquis, M.-T., 1958, Commissariat a l'Energie Atomique Bull. Inf. Sci. Tech., no. 21 , p. 1-3.

102 UCLA Radiocarbon Dates II, 1963, Radiocarbon, v. 5, p. 1-22.

103 Miami II, 1963, Radiocarbon, v. 5, p. 23-33.

104. National Physical Laboratory I, 1963, Radiocarbon, v. 5, p. 34-38.

105 Geological Survey of Canada II, 1963, Radiocarbon, v. 5, p. 39-55.

106 Texas Bio-Nuclear I, 1963, Radiocarbon, v. 5, p. 56-61.

107 Isotopes III, 1963, Radiocarbon, v. 5, p. 62-79.

108 Pennsylvania VI, 1963, Radiocarbon, v. 5, p. 82-103.

109 British Museum IV, 1963, Radiocarbon, v. 5, p. 104-108.

110 Gakushuin II, 1963, Radiocarbon, v. 5, p. 109-117.

111 New Zealand I-V, 1963, Radiocarbon, v. 5, p. 118-162.

112 Groningen IV, 1963, Radiocarbon, v. 5, p. 163-202.

113 Stockholm V, 1963, Radiocarbon, v. 5, p. 203-227.

114. Michigan VIII, 1963, Radiocarbon, v. 5, p. 228-253.

115 La Jolla III, 1963, Radiocarbon, v. 5, p. 254-272.

116 Tata I, 1963, Radiocarbon, v. 5, p. 273-282.

117 Arizona IV, 1963, Radiocarbon, v. 5, p. 283-301

118 Finland II, 1963, Radiocarbon, v. 5, p. 302-304.

119 Bern III, 1963, Radiocarbon, v. 5, p. 305-311.

120 Yale VIII, 1963, Radiocarbon, v. 5, p. 312-341.

121 Michigan IX, 1964, Radiocarbon, v. 6, p. 1.24.

122 National Physical Laboratory II, 1964, Radiocarbon, v. 6, p. 25-30.

123 Southern Rhodesia I, 1964, Radiocarbon, v. 6, p. 31-36.

124 USGS VII, 1964, Radiocarbon, v. 6, p. 37-76.

125 Rome II, 1964, Radiocarbon, v. 6, p. 77-90.

126 Arizona V, 1964, Radiocarbon, v. 6, p. 91-107.

127 Sharp I, 1964, Radiocarbon, v. 6, p. 108-109.

128 Finland III, 1964, Radiocarbon, v. 6, p. 110-111

129 RIKEN I, 1964, Radiocarbon, v. 6, p. 112-115.

130 Cambridge VI, 1964, Radiocarbon, v. 6, p. 116-137.

131 Texas II, 1964, Radiocarbon, v. 6, p. 138-159.

132 Louvain II, 1964, Radiocarbon, v. 6, p. 160-166.

133 Canada III, 1964, Radiocarbon, v. 6, p. 167-181.

134. Smithsonian Institution I, 1964, Radiocarbon, v. 6, p. 182-188.

135 Texas A \& M I, 1964, Radiocarbon, v. 6, p. 189-193.

136 Monaco I, 1964, Radiocarbon, v. 6, p. 194-196.

137 Gakushuin III, 1964, Radiocarbon, v. 6, p. 197-207.

138 Miami III, 1964, Radiocarbon, v. 6, p. 208-214.

139 Copenhagen VI, 1964, Radiocarbon, v. 6, p. 215-225

140 Tata II, 1964, Radiocarbon, v. 6, p. 226-232.

141 Saclay I, 1964, Radiocarbon, v. 6, p. 233-250.

142 Hannover III, 1964, Radiocarbon, v. 6, p. 251-268.

143 Isotopes IV, 1964, Radiocarbon, v. 6, p. 269-279.

144. Trondheim IV, 1964, Radiocarbon, v. 6, p. 280-290.

145 Uppsala IV, 1964, Radiocarbon, v. 6, p. 291-307.

146 Berlin I, 1964, Radiocarbon, v. 6, p. 308-317.

14.7 UCLA III, 1964, Radiocarbon, v. 6, p. 318-339.

148 Ohio Wesleyan I, 1964, Radiocarbon, v. 6, p. 340-348.

149 Groningen V, 1964, Radiocarbon, v. 6, p. 349-369.

150 Bern IV, 1965, Radiocarbon, v. 7, p. 1-9.

151 Gakushuin IV, 1965, Radiocarbon, v. 7, p. 10-23.

152 Canada IV, 1965, Radiocarbon. v. 7, p. 24-46. 
153 Geochron I, 1965, Radiocarbon, v. 7, p. 47-53.

154 IVIC I, 1965, Radiocarbon, v. 7, p. 54-65.

155 La Jolla IV, 1965, Radiocarbon, v. 7, p. 66-117.

156 Louvain III, 1965, Radiocarbon, v. 7, p. 118-122.

157 Michigan X, 1965, Radiocarbon, v. 7, p. 123-152.

158 Miami Corrections I-III, 1965, Radiocarbon, v. 7, p. 153-155.

159 National Physical Laboratory III, 1965, Radiocarbon, v. 7, p. 156-161.

160 New South Wales I, 1965, Radiocarbon, v. 7, p. 162-165.

161 Ohio Wesleyan II, 1965, Radiocarbon, v. 7, p. 166-173.

162 USDA Sedimentation Laboratory I, 1965, Radiocarbon, v. 7, p. 174-178.

163 Pennsylvania VII, 1965, Radiocarbon, v. 7, p. 179-186.

164 Pennsylvania VIII, 1965, Radiocarbon, v. 7, p. 187-199.

165 Packard I, 1965, Radiocarbon, v. 7, p. 200-204.

166 Cambridge VII, 1965, Radiocarbon, v. 7, p. 205-212.

167 Rome III, 1965, Radiocarbon, v. 7, p. 213-222.

168 Butomo, S. V., 1965, Radiocarbon, v. 7, p. 223-228.

169 Saskatchewan IV, 1965, Radiocarbon, v. 7, p. 229-235.

170 Saclay II, 1965, Radiocarbon, v. 7, p. 236-244.

171 Smithsonian Institution II, 1965, Radiocarbon, v. 7, p. 245-256.

172 Stockholm VI, 1965, Radiocarbon, v. 7, p. 257-290.

173 Tata III, 1965, Radiocarbon, v. 7, p. 291-295.

174. Texas III, 1965, Radiocarbon, v. 7, p. 296-314.

175 Uppsala V, 1965, Radiocarbon, v. 7, p. 315-330.

176 Uppsala VI, 1965, Radiocarbon, v. 7, p. 331-335.

177 UCLA IV, 1965, Radiocarbon, v. 7, p. 336-371.

178 USGS VIII, 1965, Radiocarbon, v. 7, p. 372-398.

179 Wisconsin I, 1965, Radiocarbon, v. 7, p. 399-407.

180 Saskatchewan I, 1955, Royal Soc. Canada trans., sec. 3, v. 49, p. 31-35.

181 Gif-sur-Yvette I, Radiocarbon, v. 8, p. 128-141.

182 Gif II, Radiocarbon, v. 8, p. 74-95.

183 Chard, C. S., 1962, Arctic Anthropology, v. 1, p. 84-86.

184 Chard, C. S., 1965, Arctic Anthropology, v. 3, p. 146-150. 